City University of New York (CUNY)

CUNY Academic Works

Publications and Research

City College of New York

1983

\title{
Low-mass Solitons from Fractional Charges in Quantum Chromodynamics
}

\author{
A. P. Balachandran \\ Syracuse University \\ V. Parameswaran Nair \\ City College of New York \\ N. Panchapakesan \\ Syracuse University \\ S. G. Rajeev \\ Syracuse University
}

\section{How does access to this work benefit you? Let us know!}

More information about this work at: https://academicworks.cuny.edu/cc_pubs/383

Discover additional works at: https://academicworks.cuny.edu

This work is made publicly available by the City University of New York (CUNY).

Contact: AcademicWorks@cuny.edu 


\title{
Low-mass solitons from fractional charges in quantum chromodynamics
}

\author{
A. P. Balachandran, V. P. Nair, N. Panchapakesan, ${ }^{*}$ and S. G. Rajeev \\ Physics Department, Syracuse University, Syracuse, New York 13210
}

(Received 25 March 1983)

\begin{abstract}
Slansky, Goldman, and Shaw have proposed a model to account for the observation of fractionally charged states. We show that in this model, there are expected to be several low-mass solitons (four being in the mass range $\sim 20-60 \mathrm{MeV}$ ) associated with the third homotopy group $\pi_{3}(\mathrm{SU}(3) / \mathrm{SO}(3))=\mathrm{Z}_{4}$, besides a low-mass $(\sim 30 \mathrm{MeV}) \mathrm{Z}_{2}$ monopole. Confirmation of these levels and hence of the model has important implications for Cabrera's results on the magnetic monopole. An efficient algorithm for the calculation of $\pi_{3}(G / H)$ for a general Lie group $G$ and a subgroup $H$ is developed. It is pointed out that solitons associated with the third homotopy group are predicted by some grand-unified-theory scenarios.
\end{abstract}

\section{INTRODUCTION}

Reports of the observation of fractional electric charges ${ }^{1}$ have prompted the suggestion by Slansky, Goldman, and Shaw ${ }^{2}$ that the color group $G=\mathrm{SU}(3)_{c}$ may be broken to the subgroup $H=\mathrm{SO}(3) \times \mathrm{Z}_{3}$. In analogy with standard QCD, they assume that only singlets under the gauged unbroken Lie group $\mathrm{SO}(3)$ appear as observable states. They have then argued that this model does not significantly disturb the standard low-energy phenomenology while at the same time it allows for fractionally charged states formed, for example, from two quarks.

In a previous paper, ${ }^{3}$ it has been pointed out that there is a non-Abelian $\left(\mathrm{Z}_{2}\right)$ monopole of very low mass $(\approx 30$ $\mathrm{MeV}$ ) in the model of Slansky, Goldman, and Shaw; the properties of this monopole have also been discussed. Such a monopole sector exists in this model because the second homotopy group $\pi_{2}(G / H)$ is $Z_{2}$.

In this paper, we point out that there is the possibility of further low-mass excitations in this model due to the fact that the third homotopy group $\pi_{3}(G / H)$ is $\mathrm{Z}_{4}{ }^{4}$. The masses of the lightest of such solitons are expected to be of the same order as the monopole mass while their topological "charges" $[\exp (2 \pi i / 4)]^{m}(m=1,2,3)$ are multiplicatively conserved. (The vacuum sector has $m=0$.)

Experimental search for these low-mass levels seems important both because of the interest of the model and because confirmation of the model will raise problems for Cabrera's results ${ }^{5}$ on magnetic monopoles. Briefly, the reason is as follows. Cabrera finds a magnetic charge of value $g$ which fulfills

$$
\frac{e g}{4 \pi}=\frac{1}{2},
$$

where $e$ is the value of the charge on the electron, while a naive application of Dirac's quantization condition in the presence of quarks gives, for the least value $g_{0}$ of the magnetic charge,

$$
\frac{(e / 3) g_{0}}{4 \pi}=\frac{1}{2},
$$

which contradicts (1.1). However, it is known ${ }^{6}$ that in the standard model, with its unbroken gauge symmetry $U(3)=S U(3) \times U(1) / Z_{3},(1.1)$ in fact gives the least magnetic charge even if there are free quarks; the associated monopole is a source for a long-range color "magnetic field" as well. But if the Slansky-Goldman-Shaw model is confirmed so that $\mathrm{U}(3)$ is broken, (1.1) is not possible for a stable monopole, and the least magnetic charge of a stable monopole fulfills (1.2); hence, a possible contradiction with Cabrera's results would have been established. We may note here that it is obviously easier to detect a lowmass excitation than to measure the properties of magnetic monopoles. [Incidentally, we may note here that models where $U(3)$ is broken to $U(1)$ or those with fractionally charged color singlets ${ }^{7}$ are also likely to have problems with (1.1). Models of the latter sort we know of have $S U(3)_{c} \times U(1)$ instead of $U(3)$ as the unbroken gauged symmetry group.]

In Sec. II, we recall the properties of the order parameter for the symmetry breakdown $\mathrm{SU}(3)_{c} \rightarrow \mathrm{SO}(3) \times \mathrm{Z}_{3}$ and then describe the origin of the solitons. It is emphasized that they are essentially the deformation of the $n$ vacuums $^{8}$ (for $n \neq 0 \bmod 4$ ) of the color group $\mathrm{SU}(3)_{c}$ to nonzero energy when $\mathrm{SU}(3)_{c}$ breaks down to $\mathrm{SO}(3) \times \mathrm{Z}_{3}$. In Sec. III, we study the symmetry properties of the order parameter in the solitonic sectors. We find that of the three solitonic sectors, only one admits spherical symmetry; the highest symmetry possible in the remaining sectors is axial. The Ansätze for the order parameter with the appropriate symmetries are written down. In Sec. IV, some properties of the solitons are inferred from the structure of the order parameter and other considerations; these properties are conveniently summarized in Sec. V. The masses of the lightest of these solitons are expected to be quite low, perhaps as low as $\sim 20-60 \mathrm{MeV}$. The solitons mix with gluons and it is perhaps reasonable to regard them as new sorts of glueballs. (Low-mass glueballs have also been suggested by Chakrabarty. ${ }^{9}$ ) The light solitons cannot decay with emission of known hadrons if their masses are as low as expected. The decay of one of these solitonic species into others of this species is certainly pos-

(c)1983 The American Physical Society 
sible if masses and conservation laws permit; it may also happen by tunneling effects which break topological conservation laws.

Section VI is devoted to an investigation of the possibility of solitons associated with the third homotopy group $\pi_{3}(G / H)$ during the course of the breakdown of several of the standard groups $G$ in particle physics (known to us) into standard subgroups $H$. A simple algorithm for the calculation of $\pi_{3}(G / H)$ is developed. The analysis recovers all known cases of such solitons; some new examples in grand-unified-theory (GUT) scenarios are also pointed out. This paper concludes with summarizing remarks in Sec. VII.

The possibility of solitons associated with a nontrivial third homotopy group was discussed a long time ago by Finkelstein and Misner. ${ }^{10}$ Subsequently and independently, it was pointed out by Skyrme ${ }^{11}$ that such solitons exist in the chiral model where $G=\mathrm{SU}(N) \times \mathrm{SU}(N)$ and $H=\mathrm{SU}(N)$. The third homotopy group $\pi_{3}(G / H)$ here is $\mathrm{Z}$, the additive group of integers, so that chiral solitons are characterized by an additive quantum number $T$ which behaves like baryon number $B$. This has even prompted the remarkable suggestion that $T$ has something to do with $B$ and that solitons with odd $|T|$ are fermions. This scenario and its variants have been studied in detail elsewhere. ${ }^{11-14}$ Unlike the chiral model, the model for the color breakdown $\mathrm{SU}(3)_{c} \rightarrow \mathrm{SO}(3) \times \mathrm{Z}_{3}$ is characterized by a finite $\pi_{3}$ group, namely, $Z_{4}$; we shall also see that there are other qualitative differences between these and chiral solitons.

\section{THE ORDER PARAMETER AND SOLITONS}

In the model under consideration, the color group $\boldsymbol{G}=\mathrm{SU}(3)_{c}$ is supposed to be (spontaneously or dynamically) broken to the subgroup $H=\mathrm{SO}(3) \times \mathrm{Z}_{3}$. We know from general theorems that the field $N$ which describes this pattern of symmetry breakdown has values in the space $G / H$ of left cosets. Our first task is to describe a convenient parametrization for $G / H$. Let $M$ be a symmetric $3 \times 3 \mathrm{SU}(3)$ matrix. Then it may be shown ${ }^{3}$ that $N$ may be defined by the equation

$$
M \lambda_{\alpha} M^{-1}=\lambda_{\beta} N_{\beta \alpha},
$$

where $\lambda_{\alpha}$ are the Gell-Mann matrices. It may also be shown that we can write

$$
M=g g^{T},
$$

where $g$ is any $3 \times 3$ matrix. [This is a consequence of Schur's theorem. Compare the discussion following (3.7) in Ref. 3. See also Ref. 15; the automorphism $\tau$ in that paper is to be identified with complex conjugation in the present context.]

The color group $\mathrm{SU}(3)_{c}$ acts on $g, M$, and $N$ according to the rules

$$
\begin{aligned}
& g \rightarrow s g, \\
& M \rightarrow s M s^{T}, \\
& N \rightarrow D(s) N D\left(s^{T}\right), \quad s \in \mathrm{SU}(3)_{c},
\end{aligned}
$$

where $D(s)$ is the octet representative of $s$. Note that $M$ is invariant under the right action of $\mathrm{SO}(3)$ :

$$
M \rightarrow M
$$

when

$$
g \rightarrow g h, h \in \mathrm{SO}(3) \text {. }
$$

We will impose the boundary condition

$$
N \rightarrow 1
$$

or

$$
M \rightarrow c 1 \text { as }|\overrightarrow{\mathrm{x}}|=r \rightarrow \infty
$$

(where $c^{3}=1$ ) on the fields $M$ and $N$. It is consistent with finiteness of energy for the effective Lagrangian of Sec. IV. It implies that we investigate only the nonmonopole sector. It also implies that

$$
g \rightarrow c^{2} h^{(\infty)} \in H \text { as } r \rightarrow \infty,
$$

where $h^{(\infty)}(r=\infty, \theta, \phi) \in \mathrm{SO}(3)$ is a function on the twosphere $S_{\infty}^{2}$ at infinity with polar coordinates $\theta$ and $\phi$. Since $\pi_{2}(\operatorname{SO}(3))$ is trivial, there exists a function $\hat{h}$ defined for all $x$ such that

$$
\begin{aligned}
& \widehat{h}(x) \in \mathrm{SO}(3), \\
& \widehat{h} \rightarrow h^{(\infty)} \text { as } r \rightarrow \infty .
\end{aligned}
$$

By letting

$$
g \rightarrow g c^{-2} \hat{h}^{-1},
$$

we can thus impose the boundary conditions

$$
\begin{aligned}
& g \rightarrow 1, \\
& M \rightarrow 1 \text { as } r \rightarrow \infty
\end{aligned}
$$

on $g$ and $M$ as well.

Because of the boundary conditions (2.9), we can regard the space $\mathscr{R}^{3}=\{x\}$ as compactified to the three-sphere $S^{3}$. The fields $g$ and $M$ map this $S^{3}$ into $G$ and $G / H$. The topological sectors of this model are thus labeled by $\pi_{3}(G / H)$. The latter is known to be $\mathrm{Z}_{4}:^{4}$

$$
\pi_{3}(G / H)=\mathrm{Z}_{4} \text {. }
$$

We will now give the analog of the winding-number formula to represent the elements of this $Z_{4}$.

Recall that

$$
\pi_{3}(G)=\mathrm{Z},
$$

and that the associated winding numbers are given by

$$
\begin{aligned}
t\left(g^{-1} d g\right) & =-\frac{1}{8 \pi^{2}} \epsilon_{i j k} \int d^{3} x \operatorname{Tr}\left[g^{-1} \partial_{i} g \partial_{j}\left(g^{-1} \partial_{k} g\right)+\frac{2}{3} g^{-1} \partial_{i} g g^{-1} \partial_{j} g g^{-1} \partial_{k} g\right] \\
& \equiv \frac{1}{24 \pi^{2}} \epsilon_{i j k} \int d^{3} x \operatorname{Tr}\left(g^{-1} \partial_{i} g g^{-1} \partial_{j} g g^{-1} \partial_{k} g\right)
\end{aligned}
$$


$t\left(g^{-1} d g\right)$ is normalized such that when $g$ maps $S^{3}$ into a fixed $S U(2)$ subgroup, it takes on the values $0, \pm 1, \pm 2, \ldots$ By writing

$$
\epsilon_{i j k} \operatorname{Tr}\left(g^{-1} \partial_{i} g g^{-1} \partial_{j} g g^{-1} \partial_{k} g\right)=\frac{1}{2} \epsilon_{i j k} \operatorname{Tr}\left(g^{-1} \partial_{i} g\left[g^{-1} \partial_{j} g, g^{-1} \partial_{k} g\right]\right)
$$

and noting that $\left[g^{-1} \partial_{i} g, g^{-1} \partial_{j} g\right]$ is an element of the Lie algebra, the normalization of $t$ when $g$ maps $S^{3}$ to $\mathrm{SO}(3)$ is seen to be proportional to the ratio of the traces of those $\mathrm{SO}(3)$ and $\mathrm{SU}(2)$ generators (with standard angular momentum commutation relations). These generators in terms of the $\lambda$ matrices are

$$
\lambda_{5},-\lambda_{7}, \lambda_{2}
$$

and

$$
\frac{\lambda_{1}}{2}, \frac{\lambda_{2}}{2}, \frac{\lambda_{3}}{2} .
$$

Thus,

$$
\frac{\operatorname{Tr}\left(\lambda_{5}{ }^{2}+\lambda_{7}{ }^{2}+\lambda_{2}{ }^{2}\right)}{\operatorname{Tr} \frac{1}{4}\left(\lambda_{1}{ }^{2}+\lambda_{2}{ }^{2}+\lambda_{3}{ }^{2}\right)}=4,
$$

and $t$ takes values $0, \pm 4, \pm 8, \ldots$ when $g$ maps $S^{3}$ to $S O(3)$.

Now consider the function

$$
z\left(g^{-1} d g\right)=\exp \left[\frac{2 \pi i}{4} t\left(g^{-1} d g\right)\right] .
$$

It has the following properties: (a) Since $t$ is integer valued,

$$
z \in Z_{4} \text {. }
$$

(b) If $h(x) \in \mathrm{SO}(3) \times \mathrm{Z}_{3}$, then $t\left(h^{-1} d h\right)$ is a multiple of 4 . Further, ${ }^{11,12}$

$$
t\left[(g h)^{-1} d(g h)\right]=t\left(g^{-1} d g\right)+t\left(h^{-1} d h\right) .
$$

Thus,

$$
z\left[(g h)^{-1} d(g h)\right]=z\left(g^{-1} d g\right) .
$$

This invariance of $z$ under $H$ gauge transformations means that we can also regard it as a function $\hat{z}$ of the order parameter $N$. [That is, given the field $N$, the value of $\widehat{z}(N)$ is given by $z\left(g^{-1} d g\right)$, where $g$ is any one of the fields which project down to $N$. The value of $z\left(g^{-1} d g\right)$ is independent of the ambiguity $g \rightarrow g h$ in the choice of $g$.] We can thus use $z$ or $\hat{z}$ to label the topological sectors.

The connection between these topological sectors and the $n$ vacuums $^{8}$ is as follows. When the color group $\mathrm{SU}(3)_{c}$ is unbroken, the gluon fields $W_{\mu}$ which give zero energy are classically pure gauges:

$$
W_{\mu}=g^{-1} \partial_{\mu} g
$$

These fields $g$ fall into topological classes characterized by the winding number $t\left(g^{-1} d g\right)$ and each such class gives a state $|n\rangle$ where (semiclassically) $t\left(g^{-1} d g\right)$ has the value $n$ in the state $|n\rangle$. We can also define a gauge transformation $T$ which changes the winding number by one unit:

$$
T|n\rangle=|n+1\rangle \text {. }
$$

When color is unbroken, there are instantons which change $n$ to $n \pm 1$. A better quantum approximation to the vacuum state is therefore the $\theta$ vacuum

$$
|\theta\rangle=\sum_{n} e^{i n \theta}|n\rangle,
$$

which is an eigenstate of $T$

$$
T|\theta\rangle=e^{i \theta}|\theta\rangle \text {. }
$$

The theory is periodic in $\theta$ with period $2 \pi$.

When the color group $G=\mathrm{SU}(3)_{c}$ is broken to $H=\mathrm{SO}(3) \times \mathrm{Z}_{3}$, the winding numbers of the classical vacuums and the instantons are associated with $H$. Thus, only the states

$$
|4 n\rangle
$$

correspond to the classical vacuums and are connected by instantons. The $\theta$ vacuum is now replaced by the $\phi$ vacuum,

$$
\begin{aligned}
& |\phi\rangle=\sum e^{4 i n \phi}|4 n\rangle, \\
& T^{4}|\phi\rangle=e^{4 i \phi}|\phi\rangle .
\end{aligned}
$$

The theory is periodic in $\phi$ with period $\pi / 2$. The excitations

$$
T|\phi\rangle, T^{2}|\phi\rangle, T^{3}|\phi\rangle
$$

(unlike $T|\theta\rangle, T^{2}|\theta\rangle, T^{3}|\theta\rangle$ ) are now new states, in general nondegenerate with $|\phi\rangle$. These are the solitonic states of our interest. When $\mathrm{SU}(3)_{c}$ is restored (by varying some parameter say), they become degenerate with $|\phi\rangle$ in the absence of instantons which change $|n|$ by values $\leq 3$ and get absorbed in the definition of $|\theta\rangle$ when such instantons are introduced.

When $G$ is unbroken, any two $T$ which change winding number by 1 are gauge equivalent. But when $G$ is broken, there may be several such $T$ which are not related by gauge transformations in $H$ and hence are not physically equivalent. This opens the possibility of several local minima of energy or quasistable states in each topological sector. This possibility is realized in our problem as we shall see in Sec. III.

The color group is a gauged group and the formulas (2.12) and (2.16) for $t$ and (hence) $z$ do not look manifestly gauge invariant. This defect is rectified by modifying $t\left(g^{-1} d g\right)$ and $z\left(g^{-1} d g\right)$ to

$$
t\left(W^{\prime}\right)=-\frac{1}{8 \pi^{2}} \epsilon_{i j k} \int d^{3} x \operatorname{Tr}\left(W_{i}^{\prime} \partial_{j} W_{k}^{\prime}+\frac{2}{3} W_{i}^{\prime} W_{j}^{\prime} W_{k}^{\prime}\right),
$$

$z\left(W^{\prime}\right)=\exp \left[\frac{2 \pi i}{4} t\left(W^{\prime}\right)\right]$, 
$W_{i}^{\prime}=g^{-1}\left(\partial_{i}+W_{i}\right) g$.

The field $W$ transforms under color according to $W_{i} \rightarrow s W_{i} s^{-1}+s \partial_{i} s^{-1}$ so that $W^{\prime}, t\left(W^{\prime}\right)$, and $z\left(W^{\prime}\right)$ are manifestly gauge invariant. Further, $z\left(W^{\prime}\right)$ is conserved as time evolves from $-\infty$ to $+\infty$. This may be seen from the identities

$$
\begin{aligned}
& t\left(W^{\prime}\right)=\int d^{3} x K_{0}, \\
& K_{\mu}=-\frac{1}{8 \pi^{2}} \epsilon_{\mu \nu \lambda \rho} \operatorname{Tr}\left(W_{\nu}^{\prime} \partial_{\lambda} W_{\rho}^{\prime}+\frac{2}{3} W_{\nu}^{\prime} W_{\lambda}^{\prime} W_{\rho}^{\prime}\right), \\
& \partial_{\mu} K_{\mu}=-\frac{1}{16 \pi^{2}} \operatorname{Tr} F_{\mu \nu}\left(W^{\prime}\right)^{*} F_{\mu \nu}\left(W^{\prime}\right), \\
& \left.t\left(W^{\prime}\right)\right|_{x_{0}=\infty}-\left.t\left(W^{\prime}\right)\right|_{x_{0}=-\infty} \\
& =\int d^{4} x \partial_{\mu} K_{\mu} \\
& =-\frac{1}{16 \pi^{2}} \int d^{4} x \operatorname{Tr} F_{\mu \nu}\left(W^{\prime}\right)^{*} F_{\mu \nu}\left(W^{\prime}\right)
\end{aligned}
$$

$\left[F_{\mu v}\left(W^{\prime}\right)\right.$ being the Yang-Mills tensor] and the fact that the last integral is restricted to the values $0, \pm 4, \pm 8, \ldots$.

Note that when $g \rightarrow g h, h \in H$,

$$
t\left(W^{\prime}\right) \rightarrow t\left(W^{\prime}\right)+t\left(h^{-1} d h\right)
$$

and

$$
z\left(W^{\prime}\right) \rightarrow z\left(W^{\prime}\right) .
$$

This means that $z\left(W^{\prime}\right)$ can be regarded as a function of the glue fields $W$ and the order parameter $N$. Since the Lagrangian will involve only the fields $W$ and $N$, we need this property of $z\left(W^{\prime}\right)$.

When $W^{\prime}$ is a pure gauge, $z\left(W^{\prime}\right)$ has values in the group $Z_{4}$. When $W^{\prime}$ is not a pure gauge, $z\left(W^{\prime}\right)$ can be any complex number of modulus 1 . The consequences of this fact will be discussed later.

A final set of remarks is in order before closing this section. As we pointed out before, the boundary conditions (2.9) imply that we are in the nonmonopole sector of the model. This will be the case throughout this paper. Our discussion will have to be modified in the monopole sector for the following reason: It is well known that the order parameter $\Phi$ in the monopole sector is obliged to have values in $G / H$ only on the sphere $S_{\infty}^{2}$ at $r=\infty$ and that it cannot have values in $G / H$ for all $x$. This is because (a) the image $\Phi\left(S_{\infty}^{2}\right)$ of $S_{\infty}^{2}$ under $\Phi$ in the monopole sector cannot be shrunk to a point while staying always in $G / H$, (b) the sphere $S_{\infty}^{2}$ at the spatial boundary can be contracted to a point by varying its radius to zero, and (c) the image of a point under $\Phi$ is a point. These remarks are in contradiction unless $\Phi$ takes values outside $G / H$. Thus, formulas like (2.1) and (2.2) do not apply to $\Phi$ for all $x$. Further, $\mathscr{R}^{3}$ in the monopole sector is not compactified to $S^{3}$, but rather to a manifold with boundary $S_{\infty}^{2}$. All this shows that we have to revise our considerations in the monopole sector.

\section{SYMMETRY CONSIDERATIONS}

The ground states of the distinct topological sectors with labels $\left(e^{2 \pi i / 4}\right)^{m} \in \mathrm{Z}_{4}$ are expected to be characterized by the highest possible symmetry of the fields consistent with topology. In analogy with monopoles and chiral solitons, we expect this symmetry to be spherical (in a generalized sense). As we shall now show, however, there exists no spherically symmetric Ansatz for our solitons except in the sector $\left(e^{2 \pi i / 4}\right)^{2}$; the best we can do in general is to construct axially symmetric Ansätze.

The angular momentum which defines spherical symmetry is assumed to be of the form

$$
J_{\alpha}=-i(\overrightarrow{\mathrm{x}} \times \vec{\nabla})_{\alpha}+\Lambda_{\alpha},
$$

where $\Lambda_{\alpha}$ span an $\mathrm{SU}(2)$ or an $\mathrm{SO}(3)$ subalgebra of the color algebra $\mathrm{SU}(3)_{c}$. We require that

$$
\begin{aligned}
J_{\alpha} N & \equiv-i(\overrightarrow{\mathrm{r}} \times \vec{\nabla})_{\alpha} N+d\left(\Lambda_{\alpha}\right) N+N d\left(\Lambda_{\alpha}^{T}\right) \\
& =0
\end{aligned}
$$

where $d\left(\Lambda_{\alpha}\right)$ is the octet representative of $\Lambda_{\alpha}$. From (2.1), this means

$$
\begin{aligned}
J_{\alpha} M & =-i(\overrightarrow{\mathrm{r}} \times \vec{\nabla})_{\alpha} M+\Lambda_{\alpha} M+M \Lambda_{\alpha}^{T} \\
& =0 .
\end{aligned}
$$

Letting $r \rightarrow \infty$ and using the boundary condition (2.9), we find that $\Lambda_{\alpha}$ are antisymmetric:

$$
\Lambda_{\alpha}+\Lambda_{\alpha}^{T}=0 \text {. }
$$

Therefore, $\Lambda_{\alpha}$ span the $\mathrm{SO}(3)$ subalgebra of antisymmetric matrices in SU(3):

$$
\left(\Lambda_{\alpha}\right)_{i j}=-i \epsilon_{\alpha i j} .
$$

It is convenient to construct spherically symmetric $g$ and find $M$ from (2.2). We can then easily check the topology of $M$ using $t$ and $z$ (which are expressed in terms of $g$ and not in terms of $M$ ). Note that the spherical symmetry of $g$, viz.,

$$
-i(\overrightarrow{\mathrm{r}} \times \vec{\nabla}) g+\left[\Lambda_{\alpha}, g\right]=0, \Lambda_{\alpha} \in \operatorname{SO}(3),
$$

implies the spherical symmetry of $M$ and $N$.

The general spherically symmetric form of $g$ is

$$
\begin{aligned}
g= & e^{i \psi}+i \sin \chi e^{-i \psi / 2} \Lambda \cdot \hat{x} \\
& +\left[\cos \chi e^{-i \psi / 2}-e^{i \psi}\right](\Lambda \cdot \hat{x})^{2}, \quad \hat{x} \equiv \frac{x}{r} .
\end{aligned}
$$

Here, $\psi$ and $\chi$ are functions of the radial variable $r$. [Evaluate $g$ after rotating $\hat{x}$ to $(0,0,1)$ to verify that $g \in \mathrm{SU}(3)$.] The boundary condition (2.9) requires that either

$$
\cos \chi(\infty)=1, e^{-i \psi(\infty) / 2}=1
$$

or

$$
\cos \chi(\infty)=-1, e^{-i \psi(\infty) / 2}=-1 .
$$

Since we can reduce (3.9) to (3.8) by the substitutions

$$
\begin{aligned}
& \chi(\infty)=\chi^{\prime}(\infty)+\pi, \\
& \psi(\infty)=\psi^{\prime}(\infty)+2 \pi,
\end{aligned}
$$


which together preserve the form of $g$, (3.9) can be ignored.

If $g$ is to be regular at the origin, the coefficients of $(\Lambda \cdot \hat{x})$ and $(\Lambda \cdot \hat{x})^{2}$ must vanish there. Thus, we require also that either

$$
\cos \chi(0)=-1, e^{-i \psi(0) / 2}=-\left(e^{2 \pi i / 3}\right)^{k}
$$

or

$$
\cos \chi(0)=1, e^{-i \psi(0) / 2}=\left(e^{2 \pi i / 3}\right)^{k^{\prime}},
$$

where $k, k^{\prime}$ are integers.

But one can show that (3.12) gives trivial topology and hence may be ignored. For let

$$
h=1+i \sin \chi \Lambda \cdot \hat{x}+(\cos \chi-1)(\Lambda \cdot \hat{x})^{2} .
$$

One can verify that $h \in \mathrm{SO}(3)$ [rotate $\hat{x}$ to $(0,0,1)$ to simplify the verification]. Further, noting that $(\Lambda \cdot \hat{x})^{3}=\Lambda \cdot \hat{x}$, we find

$$
\begin{aligned}
S & =g h^{\dagger} \\
& =e^{i \psi}+\left(e^{-i \psi / 2}-e^{i \psi}\right)(\Lambda \cdot \hat{x})^{2},
\end{aligned}
$$

which is symmetric. Therefore,

$$
\begin{aligned}
t\left(S^{-1} d S\right) & =t\left(S^{*} d S\right) \\
& =t\left(S d S^{*}\right)^{*}
\end{aligned}
$$

(as $t$ is real)

$$
\begin{aligned}
& =t\left(-d S S^{*}\right) \\
& =t\left(-S^{*} d S\right) \\
& =-t\left(S^{*} d S\right) \\
& =0,
\end{aligned}
$$

and [in view of (2.18)]

$$
\begin{aligned}
t\left(g^{-1} d g\right) & =t\left(h^{-1} d h\right) \\
& =4 n, \\
z\left(g d g^{\dagger}\right) & =1 .
\end{aligned}
$$

Thus, we need consider only (3.8) and (3.11). We now show that this combination of boundary conditions is associated with the element $\left(e^{2 \pi i / 4}\right)^{2}$ of $\mathrm{Z}_{4}$.

Note that now (3.13) is not well defined as $r \rightarrow 0$ so that $g$ cannot be reduced to a symmetric matrix as before. But if we look at $g^{2}$,

$$
\begin{aligned}
g^{2}= & e^{i \psi^{\prime}}+i \sin \chi^{\prime} e^{-i \psi^{\prime} / 2} \Lambda \cdot \hat{x} \\
& +\left(\cos \chi^{\prime} e^{-i \psi^{\prime} / 2}-e^{i \psi^{\prime}}\right)(\Lambda \cdot \hat{x})^{2}, \\
\chi^{\prime}= & 2 \chi, \quad \psi^{\prime}=2 \psi,
\end{aligned}
$$

we see that its angles fulfill (3.12). Hence,

$$
\begin{aligned}
t\left(g^{-1} d g\right) & =\frac{1}{2} t\left(g^{-2} d g^{2}\right) \\
& =\frac{1}{2} t\left(H^{-1} d H\right) \\
& =2 n,
\end{aligned}
$$

where

$$
H=1+i \sin 2 \chi \Lambda \cdot \hat{x}+(\cos 2 \chi-1)(\Lambda \cdot \hat{x})^{2} .
$$

We can also show that $n$ is odd. Let

$$
u=\cos \chi+i \tau \cdot \hat{x} \sin \chi
$$

define the $2 \times 2 \mathrm{SU}(2)$ matrix $u$. Then ${ }^{14}$

$$
t\left(u^{-1} d u\right)=\frac{1}{\pi}[\chi(0)-\chi(\infty)]
$$

and

$$
u \tau_{i} u^{\dagger}=H_{j i} \tau_{j}
$$

Therefore,

$$
\begin{aligned}
& t\left(H^{-1} d H\right)=\frac{4}{\pi}[\chi(0)-\chi(\infty)], \\
& t\left(g^{-1} d g\right)=\frac{2}{\pi}[\chi(0)-\chi(\infty)] .
\end{aligned}
$$

In view of (3.8) and (3.11), $n$ is clearly odd and

$$
z\left(g^{-1} d g\right)=-1 \text {, }
$$

as claimed.

The form of $M$ is very simple:

$$
\begin{aligned}
M & =g g^{T} \\
& =e^{2 i \psi}+\left(e^{-i \psi}-e^{2 i \psi}\right)(\Lambda \cdot \hat{x})^{2} .
\end{aligned}
$$

We see from (3.8) and (3.11) that the variation $\Delta \psi=\psi(\infty)-\psi(0)$ is given by

$$
\begin{aligned}
& \Delta \psi=2 \pi \sigma, \\
& \sigma=2 \rho+1+\frac{2}{3} k,
\end{aligned}
$$

where $\rho$ too is an integer. Thus, spherically symmetric Ansätze in the sector $\left(e^{2 \pi i / 4}\right)^{2}$ are characterized by a number $\sigma$ which assumes discrete values; such Ansätze with different $\sigma$ cannot be deformed into each other while maintaining spherical symmetry. It is unlikely, however, that $\sigma$ is a new conserved topological number, as it should be possible to deform such Ansätze into each other through configurations which do not respect spherical symmetry. We shall discuss the significance of $\sigma$ further in Sec. IV.

Axially symmetric Ansätze are easily constructed for all the topological sectors. For instance, we can take

$$
g=\left(\begin{array}{ll}
\cos \chi+i \tau \cdot \hat{x} \sin \chi & 0 \\
0 & 1
\end{array}\right]
$$

for which

$$
t\left(g^{-1} d g\right)=\frac{1}{\pi}[\chi(0)-\chi(\infty)]
$$

can take any integer value since the only constraints on $\chi$ from (2.9) and regularity at origin are

$$
\begin{aligned}
& \cos \chi(\infty)=1, \\
& \cos \chi(0)= \pm 1 .
\end{aligned}
$$

Thus, the corresponding $M$ and $N$ can be in any topological sector. Further, since 


$$
-i(\overrightarrow{\mathrm{x}} \times \vec{\nabla})_{2} g+\left[\frac{\lambda_{2}}{2}, g\right]=0,
$$

and $\lambda_{2}$ is antisymmetric, $M$ is axisymmetric:

$$
-i(\overrightarrow{\mathrm{x}} \times \vec{\nabla})_{2} M+\left[\frac{\lambda_{2}}{2}, M\right]=0 .
$$

We will not pursue these Ansätze further.

\section{THE SOLITON PROPERTIES}

The characteristic energy scale in the problem is provided by the mass ${ }^{2}$

$$
\mu_{G} \approx 20 \mathrm{MeV}
$$

of the gluons associated with the broken generators. Thus, we expect the masses $\mu_{m}$ of the lightest solitons with the quantum numbers $\left(e^{2 \pi i / 4}\right)^{m}(m \neq 0)$ to be of the same order, say, $\sim 20-60 \mathrm{MeV}$. (Of course $\mu_{0}=0$.) The length scale of the problem is also set by $\mu_{G}$; thus, these solitons are expected to be several fermis in size. A more accurate numerical estimate of the masses is difficult, as we shall see later.

Further information can be obtained from the chargeconjugation transformation

$$
g \rightarrow g^{*},
$$

which reverses $t$,

$$
\begin{aligned}
t\left(g^{-1} d g\right) & \rightarrow t\left(g^{T} d g^{*}\right) \\
& =-t\left(g^{-1} d g\right),
\end{aligned}
$$

and induces the map

$$
z\left(g^{-1} d g\right) \rightarrow z\left(g^{-1} d g\right)^{*}
$$

Thus, the $m=1$ and $3(=-1 \bmod 4)$ sectors are mutually charge conjugate. One inference from this observation is that

$$
\mu_{1}=\mu_{3} \text {. }
$$

Let us next discuss the consequences of chargeconjugation invariance in the $m=2$ sector. Note first that for each value of $\Delta \psi$ or $\sigma$, we can minimize the energy integral; we will soon see that this minimum $\mu_{2}(\sigma)$ increases rapidly with $|\sigma|$. Further, since $\Delta \psi$ reverses in sign under charge conjugation,

$$
\mu_{2}(\sigma)=\mu_{2}(-\sigma) \text {. }
$$

(The transformation $\Delta \psi \rightarrow-\Delta \psi$ corresponds to $k \rightarrow k$, $\rho \rightarrow-\rho-1$.) Further, $\sigma$ cannot vanish. Thus, before tunneling effects are considered, there are two degenerate states

$$
| \pm \sigma\rangle
$$

for each $\sigma$, with mass increasing with $|\sigma\rangle$, the lightest ones [with mass $\mu_{2}\left( \pm \frac{1}{3}\right) \equiv \mu_{2}$ ] being

$$
\left| \pm \frac{1}{3}\right\rangle \text {. }
$$

Charge conjugation interchanges the members of such a pair.

Once tunneling is considered, these states can mix and some of them can decay as well; the dominant mixing is expected to be between the charge-conjugate partners. In particular, the states $\left| \pm \frac{1}{3}\right\rangle$ will be split, the lightest one being the charge-conjugation-even combination $\left|\frac{1}{3}\right\rangle$ $+\left|-\frac{1}{3}\right\rangle$. The excited states are expected to decay into one of the states $\left|\frac{1}{3}\right\rangle \pm\left|-\frac{1}{3}\right\rangle$.

When the gluon excitations are also fully considered, there are many gluon-field configurations with the same topological quantum numbers as the solitons as we pointed out in Sec. II. The semiclassical states constructed from the order parameter will therefore mix with the gluons; we do not know, however, how to treat this effect.

We shall now indicate in greater detail how mass ratios between different levels can be estimated in the $m=2$ sector. The considerations will also reveal the problems in calculating the absolute masses.

A reasonable expression for the (static) energy from which to commence the calculation would be

$$
\begin{aligned}
E(N)= & \frac{F^{2}}{12} \int d^{3} x \operatorname{Tr}_{i} N^{\dagger} \partial_{i} N \\
& -\frac{\epsilon}{6} \int d^{3} x \operatorname{Tr}\left(\left[\partial_{i} N^{\dagger}, \partial_{j} N\right]^{2}\right),
\end{aligned}
$$

where $F$ is related to the gluon mass $\mu_{G}$ and QCD coupling constant $e$ by $^{3}$

$$
\mu_{G}{ }^{2}=2 e^{2} F \text {. }
$$

With the first term alone, dimensional analysis gives $E(N) \sim F^{2} R$ where $R$ is a characteristic length, so that the minimum of energy is zero (and corresponds to $R \rightarrow 0$ ). Since the second term goes like $R^{-1}$, with both terms, the minimum of energy is not zero. The precise form of the second term in (4.9) is suggested by the fact that such a form has played an important role in previous considerations of systems of our sort. ${ }^{11-14}$ The difficulty in calculating the absolute value of the minimum of $E(N)$ is due to the fact that there is no decent way to estimate $\epsilon$. Note that one-loop calculations do give such a term (see below).

We can write $E$ in terms of $M$ by using ${ }^{3}$

$$
\begin{aligned}
\operatorname{Tr}_{i} N^{\dagger} \partial_{i} N & =-\operatorname{Tr}\left(N^{\dagger} \partial_{i} N\right)^{2} \\
& =-6 \operatorname{Tr}\left(M^{\dagger} \partial_{i} M\right)^{2} \\
& =6 \operatorname{Tr}\left(\partial_{i} M^{\dagger} \partial_{i} M\right),
\end{aligned}
$$

etc. (This is because the trace of the squared length of a generator in the octet representation is six times that in the triplet representation, and $N^{\dagger} \partial_{i} N$ is Lie-algebra valued.) Thus,

$$
E(N)=6 E(M) \text {. }
$$

Substituting (3.25) and after some calculation, we find

$$
\begin{aligned}
E(N)= & F^{2}\left[3 \psi^{\prime 2}+\frac{4}{r^{2}}(1-\cos 3 \psi)\right] \\
& +\frac{144 \epsilon}{r^{2}} \psi^{\prime 2}(1-\cos 3 \psi)+\frac{8 \epsilon}{r^{4}}(1-\cos 3 \psi)
\end{aligned}
$$


We shall use the following stereographic Ansatz for $\psi$ :

$$
\begin{aligned}
& \frac{1}{2} \psi=-\sigma \theta \\
& \cos \theta=\frac{(r / R)^{2}-1}{(r / R)^{2}+1}, \quad \sin \theta=\frac{2(r / R)}{1+(r / R)^{2}} .
\end{aligned}
$$

It fulfills (3.26). We find

$$
\begin{aligned}
E(N)= & \left(3 \sigma^{2}+3|\sigma|-1\right) 16 \pi^{2} F^{2} R \\
& +\left(72 \sigma^{2}+3|\sigma|-1\right) \frac{64 \pi^{2} \epsilon}{R}, \sigma \neq 0 .
\end{aligned}
$$

It has a minimum

$\mu_{2}(\sigma)=64 \pi^{2} F\left[\epsilon\left(3 \sigma^{2}+3|\sigma|-1\right)\left(72 \sigma^{2}+3|\sigma|-1\right)\right]^{1 / 2}$.

The first two levels are given by $\sigma=\frac{1}{3}$ and 1 . The ratio of their masses is

$$
\frac{\mu_{2}( \pm 1)}{\mu_{2}\left( \pm \frac{1}{3}\right)}=\frac{1}{2} \sqrt{555}=11.78
$$

which is substantial.

For orientation purposes, consider the value of $\epsilon$ found in a related one-loop calculation ${ }^{13,16}$ :

$$
\epsilon=\frac{1}{768 \pi^{2}} \ln \frac{M_{H}}{F} .
$$

Here $M_{H}$ is the mass of the Higgs field responsible for symmetry breakdown. The value of $\mu_{2}(\sigma)$ is significantly affected by whether $M_{H}$ is, say, $F$ or $2 F$; however, we have no good estimate for $M_{H}$. A reliable number for $\mu_{2}(\sigma)$ is thus difficult.

It is interesting to note that spherically symmetrical excitations are present in the topologically trivial sector. In this sector, (3.11) is replaced by (3.12) and (3.26) by

$$
\sigma=2 \rho+k^{\prime}
$$

The formula (4.15) is not valid for $\sigma=0$. However, we can see directly that $\psi \equiv 0$ has $\sigma=0$ and gives zero energy so that $\mu_{0}=0$. The masses $\mu_{0}(\sigma)$ of the excited states are given by (4.16); clearly $| \pm \sigma\rangle$ are degenerate. The lightest of these have $|\sigma|=\frac{2}{3}$ and a mass

$$
\mu_{0}\left( \pm \frac{2}{3} \simeq\right)\left(\frac{231}{8}\right)^{1 / 2} \mu_{2} \simeq 5 \mu_{2}
$$

The previous remarks about mixing, etc., also apply here.

Elsewhere, ${ }^{14}$ while discussing chiral solitons, it has been pointed out that the conservation of topological quantum numbers associated with $\pi_{3}(G / H)$ can be spoiled by tunneling effects. The qualitative aspects of these arguments are readily adapted to the present model as well. However, the method of that paper for the estimation of the tunneling amplitude does not work here due essentially to the very different nature of the conservation laws in the two models. Unfortunately, we have no new method to estimate this amplitude in the present problem.

\section{SUMMARY OF SOLITONIC PROPERTIES}

(1) Four solitonic states are expected in the mass range 20-60 MeV. They are characterized by the multiplicative quantum number $[\exp (2 \pi i / 4)]^{m}(m \neq 0)$. Two of the levels have $m=1$ and 3 ; charge conjugation interchanges them. The remaining two have $m=2$ and are, respectively, even and odd under charge conjugation. They are electrically neutral.

(2) In each of these topological sectors, we expect flavor-neutral excitations (with larger values of $|\sigma|$ ) as explained earlier. More interestingly, we also expect excitations in these sectors with flavor quantum numbers, in particular electric charge (cf. Ref. 3). All such excitations, however, will decay to the ground states, the charged ones with the emission of charged leptons or hadrons depending on the energetics.

(3) There is thus quite a lot of stable or quasistable low-mass states suggested by the model. (Recall here that the model also has $Z_{2}$ monopoles. ${ }^{3}$ ) They are not seen in low-energy accelerator experiments for the same reason that diquarks are not seen. ${ }^{2}$ Thus, there is a potential between these states which rises to a height of $200 \mathrm{GeV} / \mu_{G}$ at a distance of $200 \mathrm{fm} / \mu_{G}, \mu_{G}$ being in $\mathrm{MeV}$. It falls off as $e^{-\mu_{G} r}$ at larger distances. This potential barrier prevents their copious production at low energies. They should, however, be produced at observable levels at sufficiently high energy in $e^{+}-e^{-}$or $p-p$ collisions. Further comments on the experimental consequences of these states are contained in our discussion of the $Z_{2}$ monopoles in Ref. 3.

(4) Even if these states are copiously produced in the early Universe, their present density is expected to be negligible as is also explained in our monopole paper.

\section{SKYRME'S SOLITONS IN GUT's}

Skyrme's soliton can appear in any model where a symmetry group $G$ of the Lagrangian breaks to a subgroup $H$ and the third homotopy group $\pi_{3}(G / H)$ is nontrivial. In this section, we give a simple set of rules to calculate $\pi_{3}(G / H)$ and apply the rules to GUT's. We will first state the rules and illustrate them and finally give heuristic arguments in their support.

Let us recall that for every representation $\Gamma$ of a simple compact Lie group $G$, a non-negative integer $L$ called the index (of degree 2) of the representation $\Gamma$ can be defined. ${ }^{17,18}$ [Here "simplicity" means $G$ does not have invariant Lie subgroups. Thus, $G$ can be SU(2). The notation does not show the dependence of the index on $\Gamma$ as we shall work with a fixed $\Gamma$. Finally, note that we define the index as in Ref. 18 , it is $I(2) /($ rank of $G$ ) in the notation of Ref. 17.] For many irreducible representations of simple groups, they are tabulated in Refs. 17 and 18; the index of a reducible representation is the sum of the indices of its irreducible components.

If

$$
G=\left(G_{1} \otimes G_{2} \otimes \cdots \otimes G_{n} \otimes A\right) / D
$$


where $G_{i}$ are simple, $A$ is Abelian, and $D$ discrete, the indices of $\Gamma$ for the groups $G_{i}$ can be denoted by $L_{i}$, and the index of $\Gamma$ for $G$ is then the vector

$$
\left(L_{1}, L_{2}, \ldots, L_{n}\right) \text {. }
$$

If

$$
H=\left(H_{1} \otimes H_{2} \otimes \cdots \otimes H_{k} \otimes A^{\prime}\right) / D^{\prime}
$$

(where $H_{i}$ are simple, $A^{\prime}$ is Abelian, and $D^{\prime}$ discrete) is a subgroup of such a $G$, we can of course display the index of $H$ in terms of those for $H_{i}$ as we did for $G$. This is, however, not convenient for us [essentially because $H_{i}$ may not be the subgroup of any $G_{j}$ as when $G$ is the chiral group $\mathrm{SU}(N) \times \mathrm{SU}(N)$ and $H$ is the diagonal $\mathrm{SU}(N)]$, and we choose to define the subgroup index $l$ in a different way. (If $G$ is simple, this definition is identical to the preceding definition.) If $\underline{G}, \underline{G}_{i}$ and $\underline{H}_{i}$ denote the Lie algebras of the corresponding groups, we can write

$$
\begin{aligned}
& \underline{H}_{i}=\underset{j}{\oplus} \underline{H}_{i}^{(j)}, \\
& \underline{H}_{i}^{(j)} \subseteq \underline{G}_{j},
\end{aligned}
$$

where $\underline{H}_{i}^{(j)}$ is either isomorphic to $\underline{H}_{i}$ or $\{0\}$. If the index for the associated simple group $H_{i}^{(j)}$ in $\Gamma$ is $l_{i}^{(j)}$, then the index $l_{i}$ of $H_{i}$ is the $n$-tuple

$$
\left(l_{i}^{(1)}, l_{i}^{(2)}, l_{i}^{(3)}, \ldots, l_{i}^{(n)}\right),
$$

while the index $l$ of $H$ is the collection

$$
\left(l_{1}, l_{2}, \ldots, l_{k}\right)
$$

of such $n$-tuples.

In the rules below, $\Gamma$ can be any representation of $G$ provided the associated representation of the Lie algebra $\underline{G}$ is faithful; the results for $\pi_{3}(G / H)$ are independent of this choice. Thus, we can choose $\Gamma$ conveniently subject to the stated restriction.

(1) If $G$ and $H$ are simple, then

$$
\pi_{3}(G / H)=\mathrm{Z}_{M}, \quad M=\frac{l}{L} .
$$

(2) If $G$ is simple and $H$ is of the form (6.3), then

$$
\pi_{3}(G / H)=\mathrm{Z} \text { mod every } \frac{l_{i}}{L} \text {. }
$$

(In this group, integers which differ by any $l_{i} / L$ are identified. The group operation is addition mod every $l_{i} / L$.)

(3) If $G$ and $H$ are both not simple, but of the forms (6.1) and (6.3), then $\pi_{3}(G / H)$ consists of $n$-tuples of the form

$$
\sigma=\left(\sigma_{1}, \sigma_{2}, \sigma_{3}, \ldots, \sigma_{n}\right) \bmod \text { every } \hat{l}_{i},
$$

where $\sigma_{i}$ are integers and

$$
\widehat{l_{i}}=\left[\frac{l_{i}^{(1)}}{L_{1}}, \frac{l_{i}^{(2)}}{L_{2}}, \frac{l_{i}^{(3)}}{L_{3}}, \ldots, \frac{l_{i}^{(n)}}{L_{n}}\right] .
$$

The group composition law is

$$
\sigma+\sigma^{\prime} \bmod \text { every } \hat{l}_{i} \text {. }
$$

As a first example, consider the symmetry breakdown $G=\mathrm{SU}(3) \rightarrow H=\mathrm{SO}(3) \times \mathrm{Z}_{3}$. Let $\Gamma$ be the threedimensional irreducible representation of $\mathrm{SU}(3)$. It remains irreducible under $\mathrm{SO}(3) \times \mathrm{Z}_{3}$. From pages 26 and 13 of Ref. 17 (with $a=2$ ), we see that $L=1$ and $l=4$ so that $\pi_{3}\left(\mathrm{SU}(3) / \mathrm{SO}(3) \times \mathrm{Z}_{3}\right)=\mathrm{Z}_{4}$.

As a second example, let $G$ be the chiral group $\mathrm{SU}(6) \times \mathrm{SU}(6)$ and $H$ be the diagonal subgroup $\mathrm{SU}(6)$. Here, since $G / H$ is homeomorphic to $S U(6)$ as a manifold and $\pi_{3}$ of any simple group is $Z$, we know that $\pi_{3}(G / H)=\mathrm{Z}$. We get the same result by the preceding rules. For let $\Gamma=(6,1)+(1,6)$. From p. 32 of Ref. 17, we see that $L_{1}=1=L_{2}$ while, since $H^{(1)}, H^{(2)}$ [in the notation of (6.4)] are $\mathrm{SU}(6), l^{(1)}$, and $l^{(2)}$ are 1 as well. Thus, $\pi_{3}(G / H)=\left\{\left(\sigma_{1}, \sigma_{2}\right) \bmod (1,1)\right\}=\left\{\left(\sigma_{1}, 0\right)\right\}=\mathbf{Z}$.

We shall now apply these ideas to the simple grand unifying groups $\mathrm{SU}(5), \mathrm{SO}(10), \mathrm{E}_{6}$, and $\mathrm{SU}(16)$, and to the semisimple grand unifying group $\mathrm{SO}(10) \times \mathrm{SO}(10)$ (Refs. 19 and 20). For $S U(5)$, we consider the chain

$$
\begin{aligned}
G \equiv \mathrm{SU}(5) \rightarrow G_{S} & \equiv \mathrm{SU}(3) \times \mathrm{SU}(2) \times \mathrm{U}(1) \\
& \rightarrow H \equiv \mathrm{SU}(3) \times \mathrm{U}(1) .
\end{aligned}
$$

The different chains for $\mathrm{SO}(10)$ are classified by Rajpoot $^{21}$; they need not be reproduced here. Examples of chains for $\mathrm{E}_{6}$ and $\mathrm{SU}(16)$ are

$$
\begin{aligned}
\mathrm{E}_{6} & \rightarrow \mathrm{SU}(3)_{c} \times \mathrm{SU}(2)_{L} \times \mathrm{U}(1)_{L} \times \mathrm{SU}(2)_{R} \times \mathrm{U}(1)_{R} \\
& \rightarrow \mathrm{SU}(3)_{c} \times \mathrm{SU}(2)_{L} \times \mathrm{U}(1) \\
& \rightarrow \mathrm{SU}(3)_{c} \times \mathrm{U}(1), \\
\mathrm{SU}(16) & \rightarrow \mathrm{SO}(10) \rightarrow \mathrm{SU}(5) \rightarrow \mathrm{SU}(3) \times \mathrm{SU}(2) \times \mathrm{U}(1) \\
& \rightarrow \mathrm{SU}(3) \times \mathrm{U}(1)
\end{aligned}
$$

while for $\mathrm{SO}(10) \times \mathrm{SO}(10)$, we can have, for instance,

$$
\mathrm{SO}(10) \times \mathrm{SO}(10) \rightarrow H^{\prime} \equiv \mathrm{SU}(4)_{\mathrm{HC}} \times \mathrm{SU}(3)_{c} \times \mathrm{U}(1)
$$

(HC denotes hypercolor).

It is adequate to show the calculations in detail for the first chain. We can take $\Gamma$ to be $5^{*}$ of $S U(5)$. Under $G_{S}$ it breaks up into $\left(3^{*}, 1, \frac{1}{3}\right)+\left(1,2,-\frac{1}{2}\right)$. The $\operatorname{SU}(5)$ index is $L=1$, while the $S U(3)$ and $S U(2)$ indices are also the same. Thus, $\pi_{3}\left(G / G_{S}\right)$ and $\pi_{3}(G / H)$ are trivial. As for $\pi_{3}\left(G_{S} / H\right)$, it is $\left\{\left(\sigma_{1}, \sigma_{2}\right) \bmod (1,0)\right\}=\left\{\left(0, \sigma_{2}\right)\right\}=\mathrm{Z}$ so that there are Skyrme solitons in this breakdown. They have been studied by Gipson and Tze. ${ }^{13}$ (See also Ref. 14.)

Continuing in this way, we can check that nontrivial $\pi_{3}$ occur in the following breakdowns:

(i) $G_{S} \rightarrow H[(6.12)], \pi_{3}\left(G_{S} / H\right)=\mathrm{Z}$.

(ii) Whenever we encounter the breakdown of SU(2)'s. Thus, for example, for the chain (6.13), $\pi_{3}$ is $\mathrm{Z}$ for the breakdown $x$; it is also a (new) $\mathrm{Z}$ for the breakdown $y$.

(iii) $\mathrm{SU}(16) \rightarrow \mathrm{SO}(10), \pi_{3}(\mathrm{SU}(16) / \mathrm{SO}(10))=\mathrm{Z}_{4}$. [Note that while the index of 16 for SU(16) is not tabulated in Refs. 17 and 18 , it is readily shown to be 1.]

Note that $\pi_{3}\left(\mathrm{SO}(10) \times \mathrm{SO}(10) / H^{\prime}\right)$ is trivial.

We shall now indicate the proofs of our rules for $\pi_{3}(G / H)$. It is well known that $\pi_{3}(G)=\mathrm{Z}$ when $G$ is sim- 
ple. ${ }^{8}$ In particular, if $g$ is a field that maps $S^{3}$ to $G$ and $[g]$ denotes its equivalence class under homotopy, then the map

$$
[g] \underset{t}{\rightarrow} t\left(g^{-1} d g\right)
$$

associates an integer to $[g]$ and exhibits the isomorphism from $\pi_{3}(G)$ to $\mathrm{Z}$. However, $t$ is not $H$-gauge invariant in view of (2.18) and is therefore not a map from $\pi_{3}(G / H)$. This problem is solved if we replace $t$ by $t^{\prime}$ where

$$
t^{\prime}([g])=t\left(g^{-1} d g\right) \bmod t\left(h^{-1} d h\right) \text { for every } h,
$$

$h$ being a field in $S^{3}$ with values in $H$. We have thus to figure out the set of values of $t\left(h^{-1} d h\right)$.

Consider first the case where $G$ and $H$ are simple. Recall that we can assume that $g$ and $h$ have values in an $\mathrm{SU}(2)$ [or $\mathrm{SO}(3)$ ] subgroup of $G$ and $H ;^{8}$ let the corresponding generators with standard angular momentum commutation relations be $J_{\alpha}$ and $L_{\alpha}$. Then, as in Sec. II [cf. (2.13)], $t\left(g^{-1} d g\right)$ is proportional to $\operatorname{Tr} J_{\alpha}^{2}$ while $t\left(h^{-1} d h\right)$ is proportional to $\operatorname{Tr} L_{\alpha}{ }^{2}$. Since $t\left(g^{-1} d g\right)$ has values $0, \pm 1, \pm 2, \ldots$, the values of $t\left(h^{-1} d h\right)$ are $\left(\operatorname{Tr} L_{\alpha}{ }^{2} / \operatorname{Tr} J_{\alpha}{ }^{2}\right)(0, \pm 1, \pm 2, \ldots)$. This observation shows two things: (a) $J_{\alpha}$ must span an $\mathrm{SU}(2)$ of $G$ for which $\operatorname{Tr} J_{\alpha}{ }^{2}$ is a minimum in the representation $\Gamma$, for if not $t\left(g^{-1} d g\right)$ will not assume all integer values. (b) $L_{\alpha}$ must span an SU(2) of $H$ for which $\operatorname{Tr} L_{\alpha}{ }^{2}$ is a minimum in the representation $\Gamma$ in order that we recover all possible integer values of $t\left(h^{-1} d h\right)$. With such a qualification on $J_{\alpha}$ and $L_{\alpha}$, it is possible to show that the ratio $\operatorname{Tr} L_{\alpha}{ }^{2} / \operatorname{Tr} J_{\alpha}{ }^{2}$ is just the ratio of the indices $l$ and $L$ for $H$ and $G$. By (6.17), the image under $t^{\prime}$ has values in $Z_{(l / L)}$ which is the result (6.7).

The "proof" is incomplete in that we shall not show that the map $t^{\prime}$ is one to one.

Rule (ii) follows from the fact that in (6.17), $h$ may have values in any $H_{i}$.

As regards the last rule, we recall that $\pi_{3}(G)=\oplus \pi_{3}\left(G_{i}\right)$ $=\mathbf{Z} \oplus \boldsymbol{Z} \oplus \cdots$. The map from

$$
\begin{aligned}
{[g] } & =\left[\left(g_{1}, g_{2}, \ldots, g_{n}\right)\right] \\
& =\left(\left[g_{1}\right],\left[g_{2}\right], \ldots,\left[g_{n}\right]\right),
\end{aligned}
$$

to $\left(\sigma_{1}, \ldots, \sigma_{n}\right)\left(\sigma_{i}=\right.$ integer $)$ is now defined by $[g] \rightarrow\left(t\left(g_{1}^{-1} d g_{1}\right), t\left(g_{2}^{-1} d g_{2}\right), \ldots, t\left(g_{n}^{-1} d g_{n}\right)\right)$

An element $h_{i}(x)$ in $H_{i}$ is of the form $\left(h_{i}^{(1)}, h_{i}^{(2)}, \ldots, h_{i}^{(n)}\right)(x)$ in view of (6.4). Our previous argument shows that the integer set $\left(\sigma_{1}, \sigma_{2}, \ldots, \sigma_{n}\right)$ can be transformed by an $H_{i}$ gauge transformation to

$$
\left(\sigma_{1}, \sigma_{2}, \ldots, \sigma_{n}\right)+\hat{l}_{i} \rho,
$$

where $\rho$ is an integer. Thus, we have Rule (iii).

\section{FINAL REMARKS}

In this paper, we have discussed the solitons associated with the third homotopy group when color breaks to $\mathrm{SO}(3) \times Z_{3}$ and in certain GUT scenarios. The properties of the colored solitons are summarized towards the end of Sec. V, while the solitons in GUT's are identified in Sec. VI. We have also developed an efficient method to calculate $\pi_{3}(G / H)$ (where $H$ is a subgroup of $G$ ) in Sec. VI.

The four $\pi_{3}$ solitons for the color-breaking scheme $\mathrm{SU}(3) \rightarrow \mathrm{SO}(3) \times \mathrm{Z}_{3}$ are expected to be of low mass (20-60 $\mathrm{MeV})$. This scheme also has a $\mathrm{Z}_{2}$ monopole of low mass $(\sim 30 \mathrm{MeV})$. It is our impression that this scheme can be ruled out if these low-mass excitations are not seen in the next generation of experiments. We have also argued in Sec. I that the observation of fractional charge and the results of Cabrera taken together may not be consistent with this scheme. Confirmation of these low-mass excitations will in turn have significant implications for those two experiments. Search for low-mass excitations is much easier than measuring the properties of a monopole. We feel therefore that such a search is important.

\section{ACKNOWLEDGMENTS}

This work was supported by the U. S. Department of Energy under Contract No. DE-AC02-76ER03533. A. P. B. wishes to thank Professor K. P. Sinha, Centre for Theoretical Studies, Indian Institute of Science, Bangalore, and N. P. wishes to thank Professor K. C. Wali, Physics Department, Syracuse University, Syracuse, for hospitality at their respective institutions while this work was in progress. We have benefited from discussions with F. Lizzi, S. Okubo, and J. Patera during the course of this work.
"Present address: Department of Physics and Astronomy, University of Delhi, Delhi 110 007, India.

${ }^{1}$ G. S. La Rue, W. M. Fairbank, and A. F. Hebard, Phys. Rev. Lett. 38, 1011 (1977); G. S. La Rue, J. D. Phillips, and W. M. Fairbank, ibid. 42, 142 (1979); 42, 1019(E) (1979); 46, 967 (1981); M. Marinelli and G. Morpurgo, Phys. Rep. 도, 161 (1982).

${ }^{2}$ R. Slansky, T. Goldman, and G. L. Shaw, Phys. Rev. Lett. $\underline{47}$, 887 (1981).

${ }^{3}$ A. P. Balachandran, V. P. Nair, and C. G. Trahern, Nucl.
Phys. B196, 413 (1982).

${ }^{4}$ Compare S. J. Avis and C. J. Isham, in Recent Developments in Gravitation, 1978, edited by M. Lévy and S. Deser (Plenum, New York, 1979).

${ }^{5}$ B. Cabrera, Phys. Rev. Lett. 48, 1378 (1982).

${ }^{6}$ E. Corrigan, D. I. Olive, D. B. Fairlie, and J. Nuyts, Nucl. Phys. B106, 475 (1976); E. Corrigan and D. Olive, ibid. B110, 237 (1976); P. Goddard and D. Olive, Rep. Prog. Phys. 41, 1357 (1978); H. M. Chan and S. T. Tsou, Nucl. Phys. B189, 364 (1981); A. E. Strominger, Commun. Nucl. Part. Phys. 11, 
149 (1983).

${ }^{7}$ A. De Rújula, R. C. Giles, and R. L. Jaffe, Phys. Rev. D $\underline{17}$, 285 (1978); A. Zee, Phys. Lett. 84B, 91 (1979); H. Goldberg, T. W. Kephart, and M. T. Vaughn, Phys. Rev. Lett. 47, 1429 (1981); L. F. Li and F. Wilczek, Phys. Lett. 107B, 64 (1981); H. Goldberg, Phys. Rev. Lett. 48, 1518 (1982).

${ }^{8} \mathrm{~S}$. Coleman, in The Whys of Subnuclear Physics, proceedings of the International School of Subnuclear Physics, Erice, 1977, edited by A. Zichichi (Plenum, New York, 1979), p. 837.

9J. Chakrabarti, Rochester reports, 1982 (unpublished).

${ }^{10}$ D. Finkelstein and C. Misner, Ann. Phys. (N.Y.) 6, 230 (1959); D. Finkelstein, J. Math. Phys. 7, 1218 (1966); D. Finkelstein and J. Rubinstein, ibid. 9, 1762 (1968).

${ }^{11}$ T. H. R. Skyrme, Proc. R. Soc. London A260, 127 (1961); Nucl. Phys. 31, 556 (1962); J. Math. Phys. 12, 1735 (1971).

12J. G. Williams, J. Math. Phys. 11, 2611 (1970); N. K. Pak and H. Ch. Tze, Ann. Phys. (N.Y.) 117, 164 (1979).

13J. M. Gipson and H. Ch. Tze, Nucl. Phys. B183, 524 (1981).
${ }^{14}$ A. P. Balachandran, V. P. Nair, S. G. Rajeev, and A. Stern, Phys. Rev. Lett. 49, 1124 (1982); 50, 1630(E) (1983); Phys. Rev. D 27, 1153 (1983); 27, 2772(E) (1983).

${ }^{15}$ A. P. Balachandran, R. Ramachandran, H. Rupertsberger, and B. S. Skagerstam, Lett. Math. Phys. 4, 79 (1980).

${ }^{16}$ D. I. Kazakov, V. N. Pervushin, and S. V. Pushkin, J. Phys. A 11, 2093 (1978); T. Appelquist and C. Bernard, Phys. Rev. D 22,200 (1980).

${ }^{17}$ W. J. McKay and J. Patera, Tables of Dimensions, Indices, and Branching Rules for Representations of Simple Lie Algebras (Marcel Dekker, New York, 1981).

${ }^{18}$ R. Slansky, Phys. Rep. 79, 1 (1981).

${ }^{19}$ P. Langacker, Phys. Rep. 72C, 185 (1981); J. C. Pati, A. Salam, and J. Strathdee, Nucl. Phys. B185, 445 (1981).

${ }^{20}$ A. Davidson, P. D. Mannheim, and K. C. Wali, Phys. Rev. D 26, 1133 (1982).

${ }^{21}$ S. Rajpoot, Phys. Rev. D 22,2244 (1980). 